\title{
Impedance Eduction in Sound Fields with Peripherally Varying Liners and Flow
}

\author{
W. R. Watson* and M. G. Jones ${ }^{\dagger}$ \\ NASA Langley Research Center, Hampton, Virginia 23681-2199
}

\begin{abstract}
A two-dimensional impedance eduction theory is extended to three-dimensional sound fields and peripherally varying duct liners. The approach is to first measure the acoustic pressure field at a series of flush-mounted wall microphones located around the periphery of the flow duct. The numerical solution for the acoustic pressure field at these microphones is also obtained by solving the three-dimensional convected Helmholtz equation using the finite element method. A quadratic objective function based on the difference between the measured and finite element solution is constructed and the unknown impedance function is obtained by minimizing this objective function. Impedance spectra educed for two uniform-structure liners (a wire-mesh and a conventional liner) and a hard-soft-hard peripherally varying liner (for which the soft segment is that of the conventional liner) are presented. Results are presented at three mean flow Mach numbers and fourteen sound source frequencies. The impedance spectra of the uniform-structure liners are also computed using a twodimensional impedance eduction theory. The primary conclusions of the study are: 1) when measured data is used with the uniform-structure liners, the three-dimensional theory reproduces the same impedance spectra as the two-dimensional theory except for frequencies corresponding to very low or very high liner attenuation, and 2) good agreement between the educed impedance spectra of the uniform structure conventional liner and the soft segment of the peripherally varying liner is obtained.
\end{abstract}

\section{Nomenclature}

$\mathcal{A}, K, \mathcal{P} \quad$ complex mode coefficient, axial propagation constant, normal acoustic pressure mode

$c_{0}, \rho_{0}, M_{0} \quad$ speed of sound in duct, mean static density, uniform flow Mach number in axial direction

$d, L, H, W \quad$ depth of test liner, axial location of duct termination, height of duct, width of duct

$f, \lambda \quad$ excitation frequency of sound wave, wavelength of sound wave

$i, k \quad$ unit imaginary number $(\sqrt{-1})$, free space wavenumber $\left(2 \pi f / c_{0}\right)$

$L_{1}, L_{2} \quad$ axial location of leading edge of test liner, axial location of trailing edge of test liner

$n u, n l, n d \quad$ number of flush mounted wall microphones in upstream hard wall section, liner test section,

$\vec{\nabla} \quad$ and downstream hard wall section, respectively

$p, \vec{\nabla} \quad$ 3D acoustic pressure field, 3D gradient operator

$x, y, z, t \quad$ vertical coordinate, horizontal coordinate, axial coordinate, time

$\zeta, \beta \quad$ normalized impedance, normalized admittance

\|\|$, \bullet, \vec{n} \quad$ absolute value of complex quantity, vector dot product, outward unit normal to surface

Subscripts:

$m, n$

$\mathrm{s}, \mathrm{e}$ microphone counter, mode order section, and downstream hard wall section respectively

FEM, Meas

Superscripts:

$u, l, d$ source plane quantity, exit plane quantity quantity computed from finite element analysis, quantity measured using wall microphones $u, l, d$

quantity in upstream hard wall section, liner test section, and downstream hard wall section

${ }^{*}$ Senior Research Scientist, Research Directorate, Computational AeroSciences Branch, Liner Physics Group, Mail Stop 128, (757) 864-5290, Email: Willie.R.Watson@NASA.Gov, Associate Fellow AIAA.

${ }^{\dagger}$ Senior Research Scientist, Research Directorate, Structural Acoustics Branch, Liner Physics Group, Mail Stop 463, (757) 864-5272, Email: Michael.G.Jones@NASA.Gov, Associate Fellow AIAA. 


\section{Introduction}

For over 30 years, the NASA Langley Research Center has invested a significant effort in the development of indirect impedance prediction tools and experimental rigs for the evaluation of acoustic liner impedance. ${ }^{1-13}$ This has included the development of prediction tools to account for the effects of both uniform and shear flow profiles in the duct. These tools have been successfully applied to uniform impedance samples installed in the NASA Langley Grazing Flow Impedance Tube (GFIT) and its predecessor, the Grazing Incidence Tube (GIT). These impedance eduction tools may be further divided into non-modal methods that are based on the conventional finite element method (FEM) $)^{1,2,4,5,13}$ and the single mode methods (SMM) $)^{3,6-9,11-13}$ that are based on single mode analysis. All of these methods have the restriction that they are only applicable to a rectangular waveguide with rigid sidewalls and a uniform test sample inserted into the top or bottom wall. They each have the additional restriction that the sound field in the coordinate direction perpendicular to the rigid sidewalls is independent of that coordinate direction.

In a recent paper, ${ }^{14}$ the SMM was extended to impedance eduction in a large-scale duct such as the NASA Langley Curved Duct Test Rig (CDTR) where nonplanar waves exist in the coordinate direction perpendicular to the rigid sidewalls. The analysis was significantly simplified and the impedance was educed without the complication of an iterative procedure as with the non-modal methods. However, this simpler method is restricted to uniform test samples and to a sound field for which a single high-order mode is dominant between the rigid sidewalls. Thus, a fully threedimensional (3D) impedance eduction method is still required to educe the impedance spectra when a higher-order mode is not dominant between the rigid sidewalls. The current paper has two objectives. The first objective is to extend the two-dimensional (2D) non-modal impedance eduction method to 3D. The 3D impedance eduction method is needed in large ducts (i.e., the CDTR), when there is a loss of control over the high-order duct modes (such as at the higher frequencies) and in smaller ducts (i.e., the GFIT) when the impedance of the upper or lower wall sample varies across the duct (such as a peripherally varying liner impedance). The second objective is to validate the 3D impedance eduction methodology with 1) synthesized data, and 2) measured GFIT data.

\section{Description of Physical Problem}

A schematic of the GFIT (where 3D data for the current study is measured) is shown in Fig. 1. The axial, vertical, and horizontal coordinates of the GFIT are $z, x$, and $y$, as shown in the figure. Here, the height and width of the duct are $H$ and $W$, respectively; and the origin of the coordinate system is in the lower left corner of the duct. The liner test section (that portion of duct between $z=L_{1}$ and $z=L_{2}$ ) contains a locally reacting test liner on the upper wall and the remainder of the duct is rigid. The unknown impedance of the test liner is normalized with the characteristic impedance, $\rho_{0} c_{0}$, of the air in the duct. Here $\rho_{0}$ and $c_{0}$ are the ambient density of the air and speed of sound, respectively, in the duct. Note that in this context, the unknown impedance function is assumed to be a function of both the axial and the horizontal coordinates, $(z, y)$. Thus unlike previous impedance eduction methods, the current method permits liner impedance that varies across the duct. Therefore the acoustic field will not be separable in the horizontal coordinate and a fully $3 \mathrm{D}$ code is required to educe the normalized impedance of the test liner. The mean flow in the duct is directed from left to right along the $z$ axis of the duct (see Fig. 1) and is assumed to be uniform with a mean flow Mach number of, $M_{0}$. As illustrated in the figure, the source and exit planes of the flow duct are located at $z=0$ and $z=L$, respectively. The acoustic pressure disturbance, $p_{s}(x, y)$, is known at the source plane; and at the exit plane, either the exit acoustic pressure, $p_{e}(x, y)$, or the normalized exit impedance, $\zeta_{e}(x, y)$, are known. The sound field is assumed to be composed of a single tone such that the time dependence of the acoustic field is of the form $e^{i 2 \pi f t}$, where $f$ is the frequency of the sound wave in Hertz, $i=\sqrt{-1}$, and $t$ is the time in seconds. Several flush mounted wall microphones are located around the periphery of the duct. There are $n u$ microphones in the hard-wall section upstream of the liner, $n l$ microphones in the liner test section, and $n d$ microphones in the hard-wall section downstream of the liner. In previous studies the sound field in the liner test section is 2D so that the microphones are flush mounted into the bottom wall of the duct in a linear array. However in this study the microphones are installed around the periphery of the duct so that a 3D acoustic pressure field can be measured. The problem at hand is: given the 3D acoustic pressure measurements at the microphone locations, determine the unknown, normalized impedance function, $\zeta(z, y)$, of the test liner. 


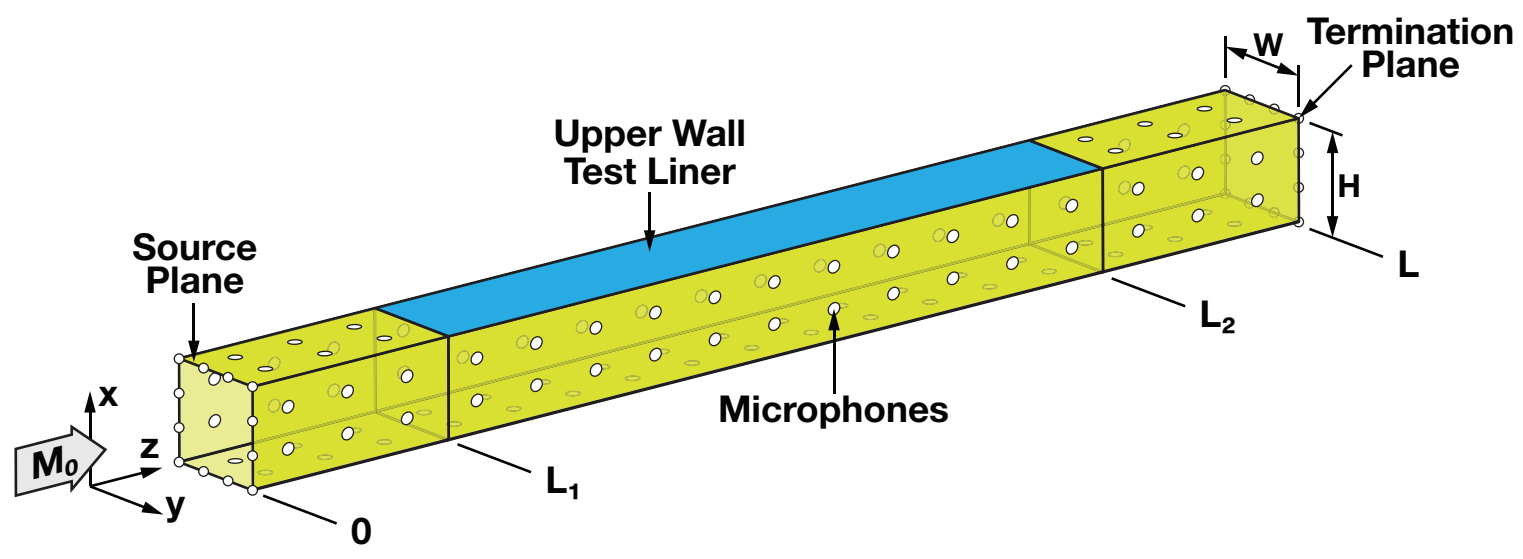

Figure 1. Schematic of the LaRC grazing flow impedance tube (GFIT) flow duct and Cartesian coordinate system.

\section{Governing Differential Equations and Boundary Conditions}

The equation that describes the propagation of linear, steady-state, acoustic pressure disturbances within the 3D duct depicted in Fig. 1 is the convected Helmholtz's equation ${ }^{2}$

$$
\left(1-M_{0}^{2}\right) \frac{\partial^{2} p(z, x, y)}{\partial z^{2}}+\frac{\partial^{2} p(z, x, y)}{\partial x^{2}}+\frac{\partial^{2} p(z, x, y)}{\partial y^{2}}-2 i k M_{0} \frac{\partial p(z, x, y)}{\partial z}+k^{2} p(z, x, y)=0
$$

where $p(z, x, y)$ is the acoustic pressure field, and $k=\frac{2 \pi f}{c_{0}}$ is the free space wave number in the duct. Across the source plane of the duct $(z=0)$ the sound source pressure is known

$$
p(0, x, y)=p_{s}(x, y)
$$

whereas at the exit plane $(z=L)$ the exit pressure is also known

$$
p(L, x, y)=p_{e}(x, y)
$$

As implied earlier, a normalized exit impedance may also be prescribed. In this case the exit plane boundary condition (BC) given in Eq. (3) is replaced with the exit impedance BC

$$
\vec{\nabla} p \bullet \vec{n}=i k \bar{\beta} p, \quad \bar{\beta}=\frac{\beta_{e}+\left(M_{0} / i k\right) \frac{\partial \beta_{e}}{\partial z}}{1+M_{0} \beta_{e}}, \quad \beta_{e}=\frac{1}{\zeta_{e}}
$$

where $\vec{n}$ is the outward pointing normal to the exit plane boundary. Note that in Eqs. (2)-(4) the source pressure, $p_{s}(x, y)$, and exit pressure, $p_{e}(x, y)$ as well as the exit impedance, $\zeta_{e}(x, y)$, are functions of position along their respective boundary. Finally, the lower and sidewalls of the duct are rigid

$$
\frac{\partial p(z, 0, y)}{\partial x}=\frac{\partial p(z, x, 0)}{\partial y}=\frac{\partial p(z, x, W)}{\partial y}=0
$$

and the test liner is assumed locally reacting so that the appropriate wall impedance boundary condition ${ }^{16}$ is

$$
-\frac{\partial p(H, y, z)}{\partial x}=i k \frac{p(H, y, z)}{\zeta(z, y)}+2 M_{0} \frac{\partial}{\partial z}\left[\frac{p(H, y, z)}{\zeta(z, y)}\right]+\frac{M_{0}^{2}}{i k} \frac{\partial^{2}}{\partial z^{2}}\left[\frac{p(H, y, z)}{\zeta(z, y)}\right]
$$

where the normalized, locally reacting wall impedance function, $\zeta(z, y)$, is a function of both the axial coordinate, $z$, and the horizontal coordinate, $y$. Given the 3D acoustic pressure measurements in the duct, Eqs. (1)-(6) constitute a well-posed boundary value problem that can be solved to determine the unique, ${ }^{15}$ unknown, normalized impedance function, $\zeta(z, y)$, of the test liner. This process is referred to as impedance eduction in the duct acoustics literature. The proposed 3D impedance eduction method presented in this paper is described in the following section. 


\section{The 3D Impedance Eduction Method}

The proposed 3D impedance eduction method parallels that used in the 2D impedance eduction method ${ }^{2}$ and is summarized as follows:

1. Three-dimensional acoustic pressure measurements are acquired at the $(n u+n l+n d)$ flush mounted wall microphones located around the periphery of the duct (see Fig. 1)

2. A guess for the normalized impedance function, $\zeta(z, y)$, is provided

3. Equations (1)-(6) are solved with a 3D finite element method (FEM) that employs cubic Hermite polynomial basis functions that is nominally fourth order accurate. The details of the 3D FEM are rather lengthy and are not presented in this paper. However, it is a straight forward extension of the 2D method that has been presented in a number of previous papers ${ }^{1,2,4,5}$

4. The FEM solution is used to construct the objective function

$$
\begin{aligned}
F(\zeta)= & \frac{1}{n u} \sum_{m=1}^{n u}\left\|p_{\text {Meas }}^{u}\left(z_{m}, x_{m}, y_{m}\right)-p_{\text {FEM }}^{u}\left(z_{m}, x_{m}, y_{m}\right)\right\|+\frac{1}{n l} \sum_{m=1}^{n l}\left\|p_{\text {Meas }}^{l}\left(z_{m}, x_{m}, y_{m}\right)-p_{\text {FEM }}^{l}\left(z_{m}, x_{m}, y_{m}\right)\right\| \\
& +\frac{1}{n d} \sum_{m=1}^{n d}\left\|p_{\text {Meas }}^{d}\left(z_{m}, x_{m}, y_{m}\right)-p_{\text {FEM }}^{d}\left(z_{m}, x_{m}, y_{m}\right)\right\|
\end{aligned}
$$

In Eq. (7), the subscripts "FEM" and "Meas" denote the acoustic pressure field computed at the microphone location using the FEM and those measured with the flush mounted wall microphones, respectively, |||| denotes the absolute value of a complex quantity, and the superscripts $u, l$, and $d$ denote the hard-wall section upstream of the liner, the liner test section, and the hard-wall section downstream of the liner, respectively

5. The impedance function, $\zeta$, that is the global minimum of $F(\zeta)$ is taken as the unknown normalized impedance function of the test liner. This minimum is obtained using the Stewart's Adaptation of the Davidon-FletcherPowell (SDFP) optimization algorithm. ${ }^{18}$

\section{Mode Solution for Acquiring Synthesized Data}

Exact solutions for the acoustic pressure field in the duct for arbitrary functional forms of the normalized impedance function, $\zeta(z, y)$, have yet to be found. However, a modal solution exists in the liner test section when the impedance function, $\zeta$, is constant (i.e., infinitely lined duct). This mode solution is used to obtain synthesized data for validating the 3D impedance eduction methodology. For the duct depicted in Fig. 1, this mode solution is obtained by assuming that the 3D acoustic pressure field in the liner test section can be expanded into a series of normal acoustic pressure modes

$$
p(z, x, y)=\sum_{n=1}^{\infty} \mathcal{A}_{n} e^{-i K_{n} z} \mathcal{P}_{n}(x, y)
$$

where, $\mathcal{P}_{n}$, is the normal acoustic pressure mode, $K_{n}$, is the axial propagation constant, and, $\mathcal{A}_{n}$, is the complex mode coefficient for the mode expansion. In Eq. (8) the right and left moving duct modes are associated with a $K_{n}$ possessing positive and negative imaginary parts, respectively. If $K_{n}$ is wholly real (as with a cuton mode in a hard-wall duct) then the left and right moving duct modes have a positive and a negative $K_{n}$, respectively. The normal acoustic pressure modes, $\mathcal{P}_{n}$, and axial propagation constants, $K_{n}$, are obtained numerically by a matrix method similar to that developed by Watson and Lansing. ${ }^{17}$ However, the method used here has been extended to include the effects of uniform mean flow. Once the normal acoustic pressure modes and axial propagation constants are obtained using the mode method, the mode expansion given in Eq. (8) is evaluated at each microphone location around the duct periphery to obtain synthesized data to validate the 3D impedance eduction procedure.

\section{Test Liners}

Three locally reacting liners are tested is this paper. Two of the liners are uniform in structure and the other liner is a three-segment peripherally varying liner (i.e., its liner impedance varies across the top wall of the duct). Each test liner is described further in the following two subsections. 


\section{A. Uniform liners}

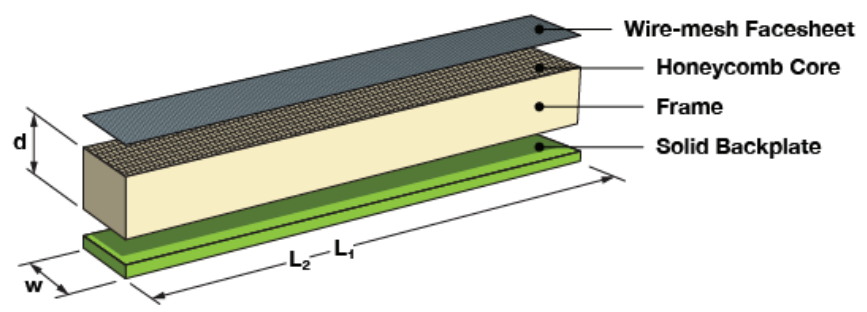

(a) Wire-Mesh Liner

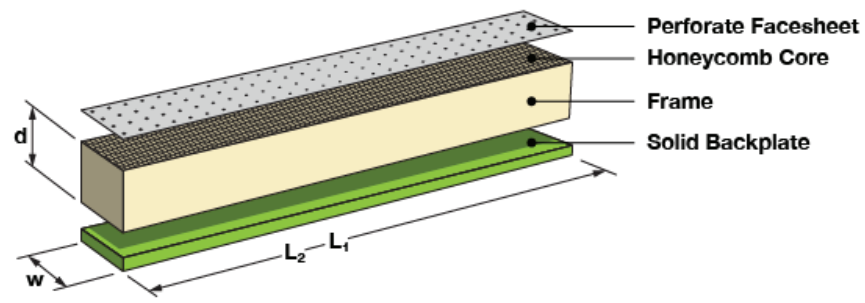

(b) Conventional liner

Figure 2. Schematic of uniform-structure test liners.

Figure 2 is a schematic of the two uniform-structure test liners that are used in this investigation. The first uniformstructure liner [Fig. 2(a)] is referred to as the wire-mesh liner because it is constructed of a wire-mesh facesheet mounted onto a honeycomb core. This liner is $610.0 \mathrm{~mm}$ long $\left(L_{2}-L_{1}\right), 51.0 \mathrm{~mm}$ wide $(W)$, and $76.0 \mathrm{~mm}$ deep $(d)$. The wire-mesh facesheet has a flow resistance of 270 MKS Rayls and the honeycomb chambers are hexagonal shaped with a nominal diameter of $9.5 \mathrm{~mm}$. The second uniform-structure liner [Fig. 2(b)] is a $610.0 \mathrm{~mm}$ long $\left(L_{2}-L_{1}\right)$ single layer conventional liner consisting of a perforated facesheet bonded to a honeycomb core. The conventional liner has the same width as the wire-mesh liner. Although the honeycomb chambers in the conventional liner have the same hexagonal shape and nominal diameters as that of the wire-mesh liner, their depth is only half that of the wire-mesh liner $(d=38.0 \mathrm{~mm})$. The perforated facesheet of the conventional liner is $0.8 \mathrm{~mm}$ thick, its porosity is $5 \%$, and the hole diameter is $0.3 \mathrm{~mm}$. These two liners are chosen because the resistance spectra of the wire-mesh liner is expected to be nearly independent of the mean flow Mach number, whereas that of the conventional liner is expected to increase with increasing mean flow Mach number. Data acquired with these two uniform-structure liners installed in the GFIT are used to validate the $3 \mathrm{D}$ impedance eduction method.

\section{B. Three-Segmented Peripherally Varying Liners}

In addition to the tests on the two uniform-structure liners (see Fig. 2) tests are also conducted on the three-segmented peripherally varying conventional liner shown in Fig. 3. 


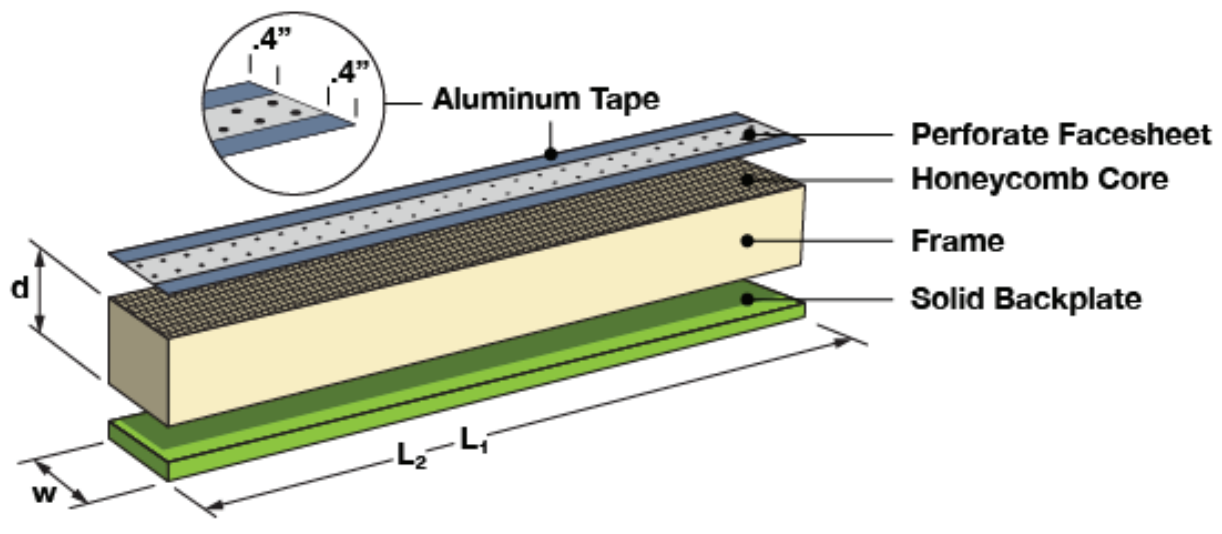

Figure 3. Schematic of three-segmented peripherally varying test liner.

The three-segmented liner is constructed by placing axial strips of aluminum tape along the left and right edges of the conventional liner. This converts the conventional liner into a hard-soft-hard peripherally varying liner whose impedance variation across the upper wall was symmetric about the duct center span. The soft portion of the liner is $3 W / 5$ units in width and the two taped sections are each $W / 5$ units in width. Note that the educed impedance of the soft segment of the peripherally varying liner should match that of the uniform-structure conventional liner.

\section{Results and Discussion}

Results presented in this section are for the GFIT geometry. The FEM solution is compared to the exact mode solution for a uniformly lined duct [Eq. (8)] and a design order convergence rate is achieved as the computational mesh is refined. The residual error in the FEM solution reduces by a factor of $2^{4}$ for each doubling of the grid. The FEM grid used for the impedance eductions is evenly spaced in each coordinate direction. There are 81 evenly spaced points in the axial direction (the $z$ direction), 26 evenly spaced points in the vertical direction (the $x$ direction), and 21 evenly spaced points in the horizontal direction (the $y$ direction). This grid is sufficiently fine to resolve all cuton waves in the hard wall sections of duct for the frequencies of interest in this study. Although the sound field in the liner test section is typically 3D, all experimental results are acquired using a plane wave as the sound source. This is achieved by keeping the sound frequency below the cuton of higher order duct modes in hard wall sections of duct.

\section{A. Uniform-Structure Wire Mesh Liner}

The wire-mesh liner [Fig. 2(a)] is inserted into the GFIT and the 2D impedance eduction code ${ }^{1,2,4,5}$ is used to establish baseline impedance spectra for this uniform-structure for a plane wave source at an incident sound pressure level (SPL) of $130 \mathrm{~dB}$. Baseline resistance [i.e., $\mathfrak{R}(\zeta)$ ] and reactance [i.e., $\mathfrak{I}(\zeta)$ ] spectra are computed for three targeted centerline Mach numbers and fourteen frequencies ranging from 0.4 to $3.0 \mathrm{kHz}$ in $0.2 \mathrm{kHz}$ increments. The targeted centerline Mach numbers for the wire-mesh liner are $M_{0}=0.0, M_{0}=0.3$, and $M_{0}=0.5$. The GFIT data used for these $2 \mathrm{D}$ eductions are obtained using a linear array of 53 lower wall microphones.

The wire-mesh liner is then used to validate the $3 \mathrm{D}$ impedance eduction code. This is achieved by using the baseline impedance spectra educed in the GFIT (from the 2D code) to obtain synthesized data for the 3D code. Synthesized data for the 3D code is acquired at the 87 microphones located around the peripherally of the duct via the mode solution given in Eq. (8). Note that the mode solution [Eq. (8)] is valid only over the length of the liner (i.e., from $z=L_{1}$ to $z=L_{2}$ ). Therefore to acquire the synthesized data, the 3D code was run with the leading edge of the liner at the source plane $(z=0)$ and the trailing edge of the liner at the duct termination $(z=L)$. Here we use synthesized data for two different nonplanar sources. The first source is the least attenuated mode with the mode coefficient, $\mathcal{A}_{1}$, set to a SPL of $130 \mathrm{~dB}$. The second source is a multimodal sound source consisting of the first and second least attenuated mode. In the multimodal sound source calculations, the mode coefficients for each of the two modes $\left(\mathcal{A}_{1}\right.$ and $\left.\mathcal{A}_{2}\right)$ are set to a level of $130 \mathrm{~dB}$. 


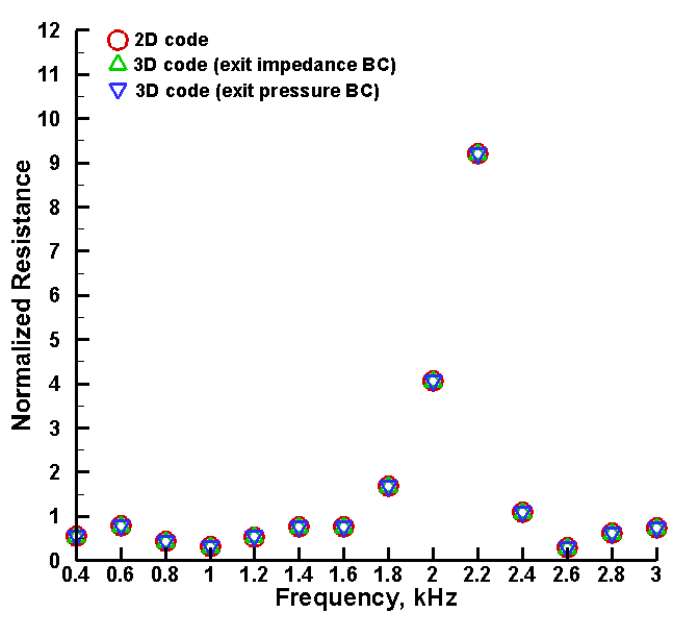

(a) Normalized Resistance

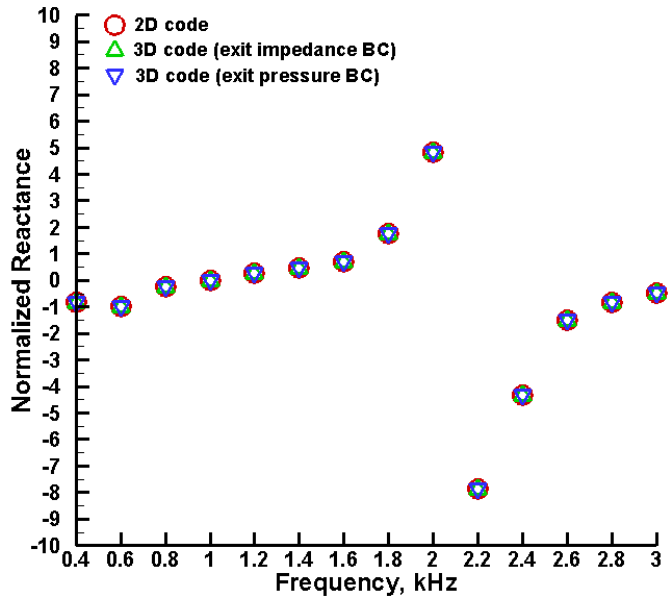

(b) Normalized Reactance

Figure 4. Comparison of educed normalized resistance and reactance spectra from 2D and 3D codes at Mach 0.5 (wire-mesh liner).

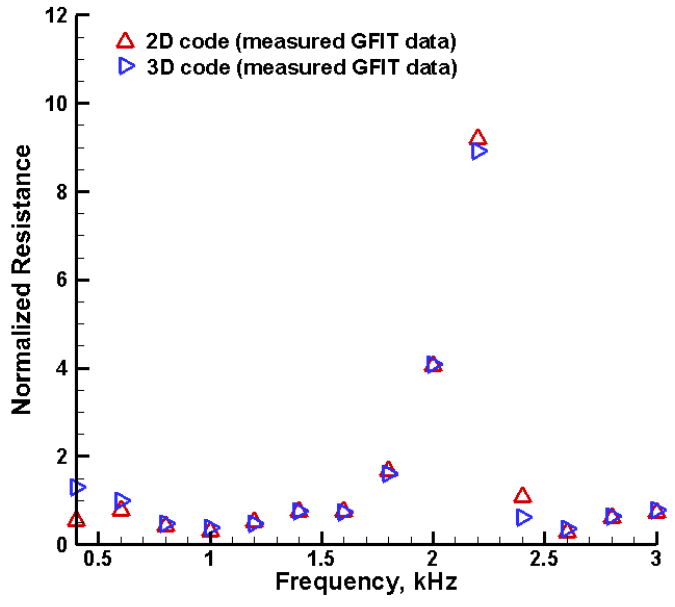

(a) Normalized Resistance

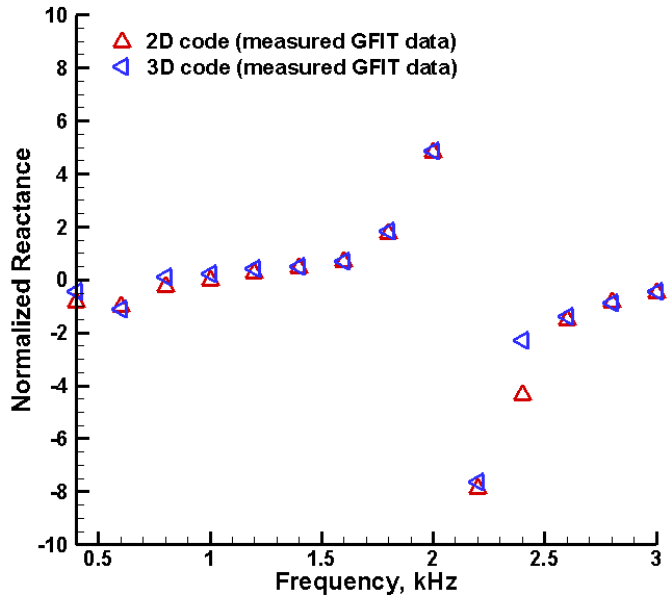

(b) Normalized Reactance

Figure 5. Effects of measurement error on the comparison of 2D and 3D impedance eduction codes at Mach 0.5 (wire-mesh liner).

Figures 4(a) and 4(b) compare the educed normalized resistance and reactance spectra educed from the 2D and $3 \mathrm{D}$ codes at a Mach number of $0.5\left(M_{0}=0.5\right)$. Recall that the $2 \mathrm{D}$ code requires both the measured GFIT data (at 53 lower wall microphones) and the GFIT geometry (i.e., $203.0 \mathrm{~mm}$ long hard-wall sections were located upstream and downstream of the liner) to educe the impedance. The 3D code uses synthesized data (acquired using the same impedance as the 2D code over the entire length of the duct). In the 3D code the data at the 87 microphones located around the duct periphery are synthesized from the exact mode solution given in Eq. (8). Impedances educed for the 3D code used the least attenuated mode as the sound source and results are shown using both exit boundary conditions (i.e., the exit impedance $\mathrm{BC}$ and the exit pressure $\mathrm{BC}$ ). As expected, the 3D code produces impedance spectra identical to that of the 2D code regardless of whether the exit pressure or exit impedance $\mathrm{BC}$ is used. Results similar to those in Figs. 4(a) and 4(b) are obtained for the multimodal sound source and for the other Mach numbers (i.e., Mach 0.0 and Mach 0.3). Such excellent comparison between the 2D and 3D code validates the $3 \mathrm{D}$ impedance eduction method for a uniform liner (at least in the presence of synthesized data). 
The results obtained from the 3D impedance eduction code with measured data as input may not be as accurate as that obtained using synthesized data. Recall that measured data contain measurement error that is not present in the synthesized data that is obtained from the exact mode solution. Therefore the measured 2D data acquired in the GFIT (for the wire-mesh liner) was processed through the 3D impedance eduction code to assess the effects of measurement error. This assessment was made using only the exit pressure BC and the measured data from the 53 lower wall microphones is used to educe the impedance in each code. Normalized resistance and reactance spectra computed from the codes are compared in Figs. 5(a) and 5(b), respectively. Observe that the educed normalized resistance and reactance predicted from the $2 \mathrm{D}$ and $3 \mathrm{D}$ codes (Figs. 4) are in very good agreement except at the low frequency end of the spectra (i.e., $f \approx 0.4 \mathrm{kHz}$ ) and near the anti-resonant frequency (i.e., $f=2.2 \mathrm{kHz}$ ). This is expected because at these frequencies the liner attenuation is the lowest. The measured liner attenuation is $1.5 \mathrm{~dB}$ at the anti-resonant frequency (i.e., $f=2.2 \mathrm{kHz}$ ) and only $3.0 \mathrm{~dB}$ at the lowest frequency $(f=0.4 \mathrm{kHz}$ ). This can be contrasted to the liner attenuation at the resonant frequency (i.e., $f=1.0 \mathrm{kHz}$ ), which is $52 \mathrm{~dB}$. Thus the accuracy of the educed impedance is reduced in that portion of the frequency spectrum where the liner attenuation is low. It should also be noted that at the lower frequency (i.e., $f=0.4 \mathrm{kHz}$ ) the wavelength, $\lambda$, of the sound wave (i.e., $\lambda=\frac{\left(1+M_{0}\right) c_{0}}{f} \approx 1285 \mathrm{~mm}$ ) exceeds the duct length $(L=1016 \mathrm{~mm})$. When the wavelength of sound is greater than the length of the liner and the length of the duct, the effects of the liner impedance discontinuities may not be adequately captured by the impedance eduction theory. The convective effects of the flow will further reduce the liner attenuation and will also tend to increase the wavelength of the sound waves relative to the liner length. Thus, the discrepancies near the low frequency end of the spectrum $(f=0.4 \mathrm{kHz})$ and near the anti-resonant frequency $(f=2.2 \mathrm{kHz})$ increase with the flow Mach number.

\section{B. Conventional Liner Study}

In addition to the tests on the wire-mesh liner, the following tests are conducted on the conventional liner:

1. The uniform-structure conventional liner [Fig. 2(b)] is inserted into the GFIT and the baseline impedance spectra is determined using the $2 \mathrm{D}$ impedance eduction code. The source is an incident $120 \mathrm{~dB}$ plane wave source and the mean flow Mach numbers are Mach 0.0, Mach 0.2, and Mach 0.3. The Mach numbers of the conventional liner were different from those of the wire-mesh liner because this conventional liner is also being tested in another aerospace laboratory for which the highest Mach number achievable is Mach 0.3. The frequencies tested for the conventional liner were identical to those of the wire-mesh liner.

2. The three-segmented hard-soft-hard peripherally varying liner (Fig. 3) for which the soft portion was the conventional liner is also tested in the GFIT. The test conditions (i.e., sound source, mean flow Mach number, and sound frequencies) match those used for the uniform-structure conventional liner. The 3D acoustic pressure field due to the three-segmented peripherally varying liner is measured using the 87 microphones that are flush mounted around the periphery of the GFIT as illustrated in Fig. 1. The impedance of the soft portion of the three-segmented peripherally varying liner is then educed using the 3D code (with the impedance of the aluminum tape set to that of a rigid wall). The impedance spectra educed for the soft segment of the peripherally varying liner is compared to that educed when using the uniform-structure conventional liner. The impedance of the soft section of the three-segmented peripherally varying liner should match that of the uniform-structure conventional liner.

Figure 6 compares the educed normalized resistance from the 2D and 3D code for the uniform-structure conventional liner. The 2D code used the 53 lower wall microphones closest to the duct center span while the 3D codes used the full array of microphones (i.e., 87 microphones) that were inserted around the periphery of the duct. At Mach 0.0 and 0.2 there is favorable agreement between the normalized resistance spectra educed from both codes. There is also a small amount of scatter in the educed resistance comparison at the high frequency end of the spectra at the highest Mach number (i.e., Mach 0.3). As expected, the resistance increases with the mean flow Mach number. The educed normalized reactance for the 2D and 3D codes are compared in Fig. 7 and they are in very good agreement. Note that the educed normalized reactance is independent of the mean flow Mach number and follows a $\cot (k d)$ behavior (as expected). There is a small amount of scatter in the reactance comparisons at the highest frequency $(f=2.6 \mathrm{kHz})$ and Mach number (Mach 0.3).

The presence of the hard-wall strips along the left and right edges of the segmented liner results in a scattering of energy into higher order horizontal modes and the generation of a 3D sound field. Consequently, the absorption characteristics of the segmented liner differs from that of the uniform-structure liner (the uniform-structure liner alone 


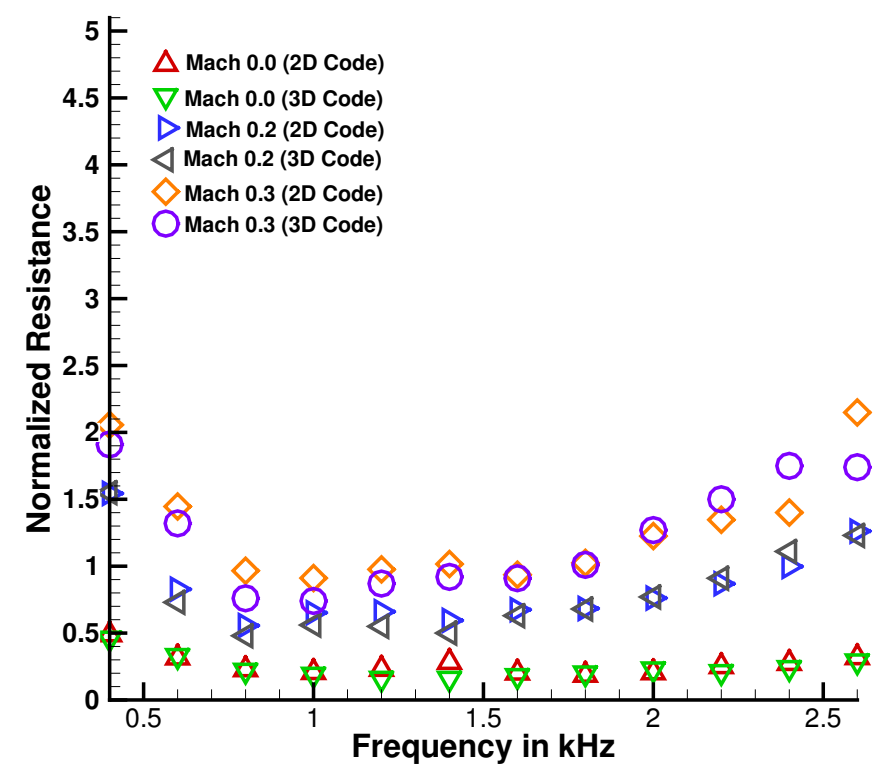

Figure 6. Comparison of educed normalized resistance spectra from 2D and 3D codes for the uniform-structure conventional liner.

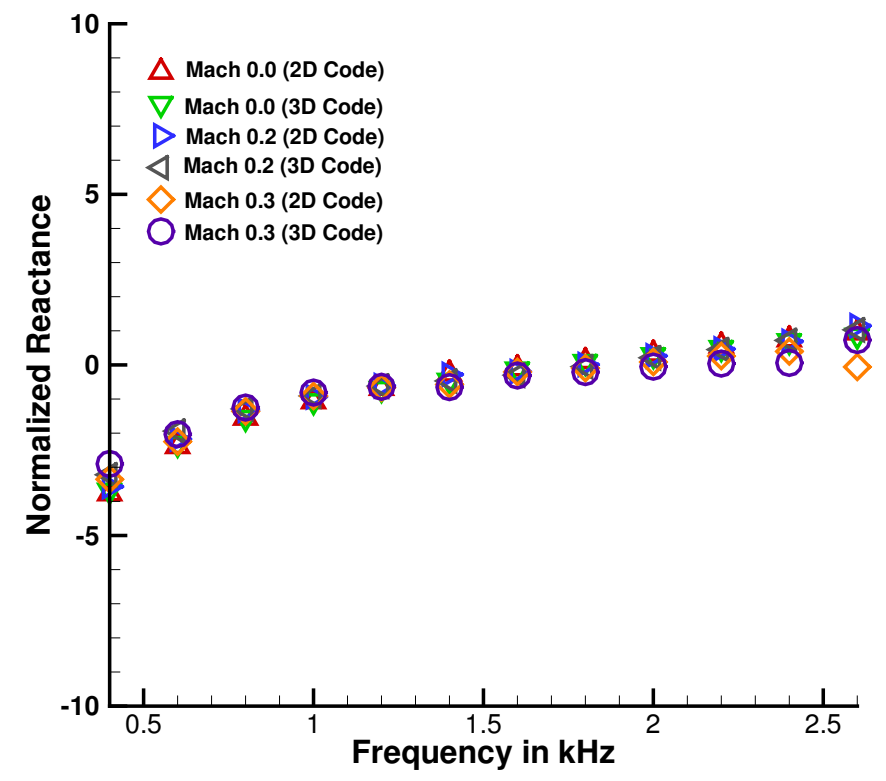

Figure 7. Comparison of educed normalized reactance spectra from 2D and 3D codes for the uniform-structure conventional liner.

cannot produce this type of scattering). Figure 8 compares the measured sound pressure level profile of the uniformstructure liner and the three-segmented peripherally varying liner at a frequency of $0.8 \mathrm{kHz}$. The leading and trailing

9 of 13 
edges of the liner are located at $z=0.2$ and $z=0.61$ meters, respectively. The acoustic pressure measurements are taken along the lower wall at the duct centerline (i.e., $y=W / 2$ ). When there is no flow (Mach 0.0), the three-segmented liner attenuates much more sound than the uniform-structure liner (Fig. 7) and there is a significant impact of the hardwall strips. As the flow Mach number increases, the uniform-structure liner becomes a much better attenuator of the sound when compared to the segmented liner (see Fig. 8). Figure 9 shows similar results at the frequency closest to the resonant frequency (i.e., $f=1.4 \mathrm{kHz}$ ) where there is a large amount of liner attenuation. Note that near the resonant frequency there is a significant impact of the hard wall strips because the SPL patterns are significantly different for the uniform-structure and the peripherally segmented liner. Observe that the uniform liner is a much better attenuator of the sound waves near the resonance frequency for all flow Mach numbers (Fig. 9).

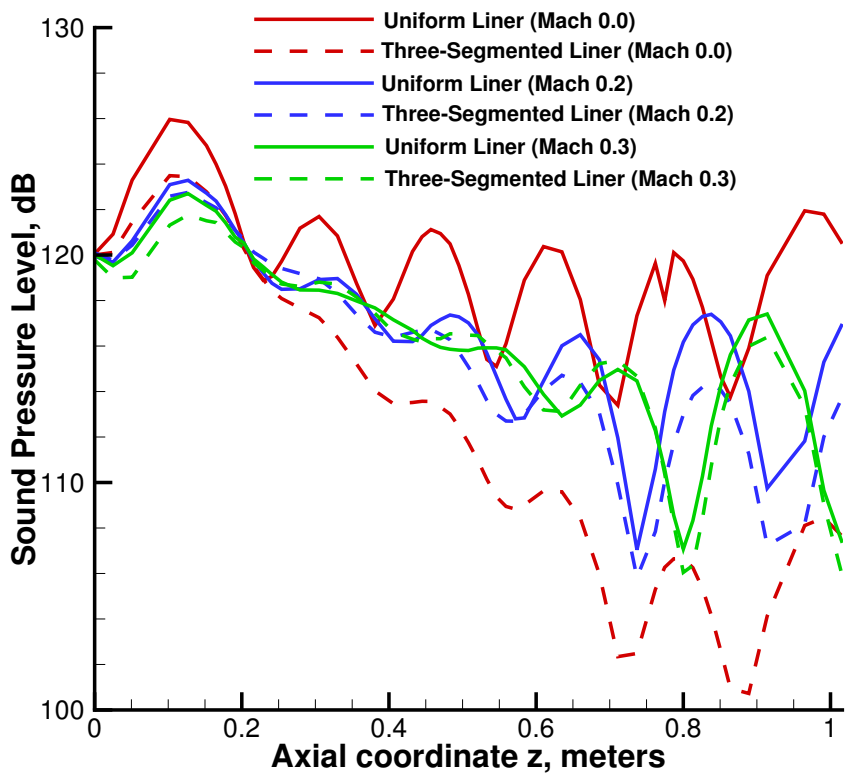

Figure 8. Comparison of measured SPL over the lower wall for the uniform and three-segmented liner at $800 \mathrm{~Hz}$. 


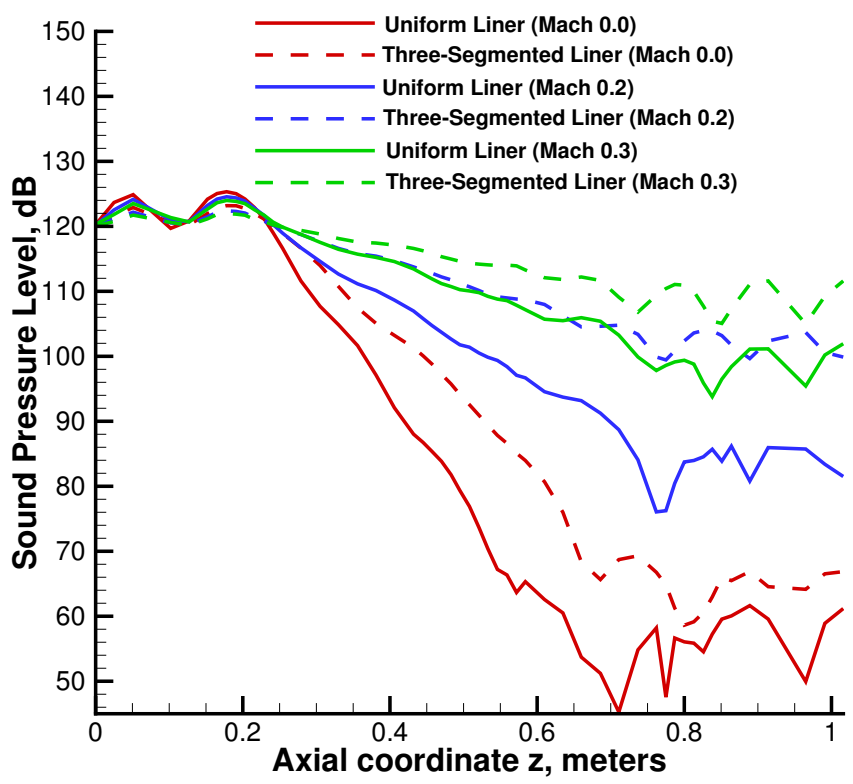

Figure 9. Comparison of measured SPL over the lower wall for the uniform and three-segmented liner at 1,400 Hz.

In this final example, all of the 87 microphones inserted around the periphery of the GFIT (Fig. 1) are used to measure the acoustic field produced by 1) the uniform-structure conventional liner, and 2) the three-segmented peripherally varying liner. These measured acoustic fields are then used to a) educe the impedance of the uniformstructure conventional liner, and to b) educe the impedance of the soft segment of the three-segmented peripherally varying liner. Note that the impedance of the soft segment of the peripherally varying liner should match that of the uniform-structure conventional liner.

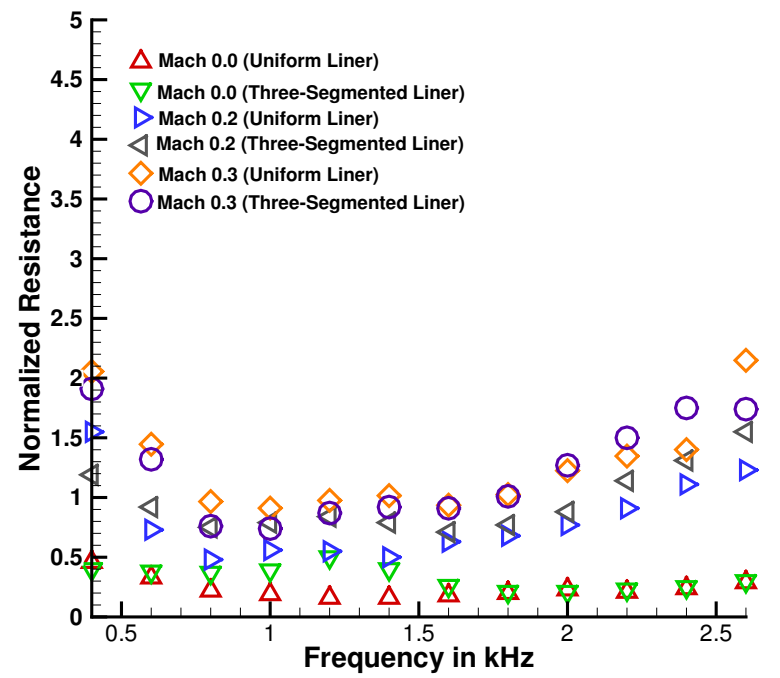

Figure 10. Comparison of the educed normalized resistance spectra for the uniform and three-segmented liner.

11 of 13 


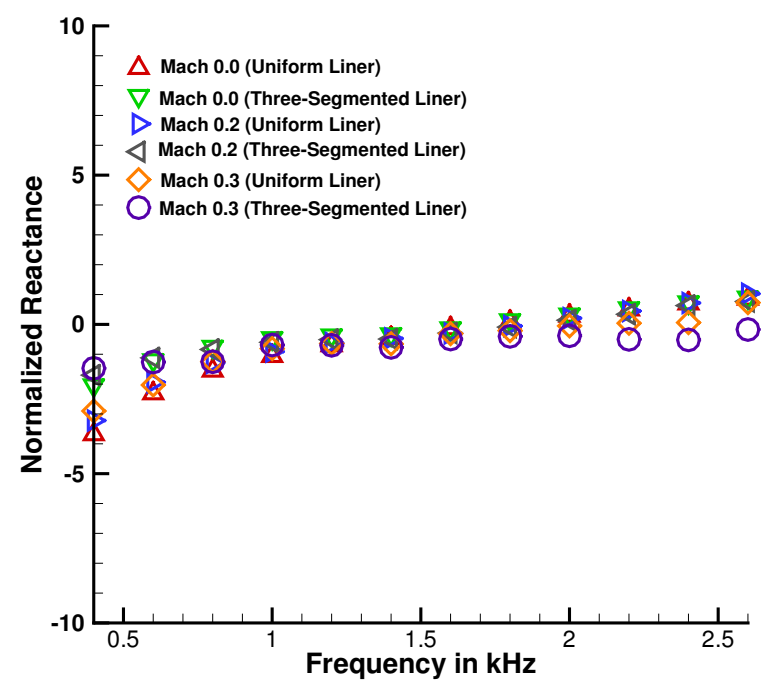

Figure 11. Comparison of the educed normalized reactance spectra for the uniform and three-segmented liner.

Educed normalized resistance spectra are shown in Fig. 10. Note that the zero flow results (the Mach 0.0 results) match extremely well with the exception of the two frequencies closes to the resonant frequency (i.e., $1.2 \mathrm{kHz}$ and $1.4 \mathrm{kHz}$ ). This is due to the large attenuation (approximately $70 \mathrm{~dB}$ was measured) near the resonant frequency. Resolving such a large attenuation is a difficult task for any numerical scheme including the FEM. As the Mach number is increased to 0.2 the comparison between the educed normalized resistance for the uniform-structure sample and the soft segment of the peripherally varying liner is still quite good (except for the two frequencies closest to the resonant frequency where the attenuation is large). The discrepancy at 0.4 and $0.8 \mathrm{kHz}$ is due to the small attenuation at those frequencies. The frequencies with the lowest attenuation were 0.4 and $0.8 \mathrm{kHz}$. For example, the attenuation of the liner is measured to be no more than $0.2 \mathrm{~dB}$ at $0.8 \mathrm{kHz}$. As the mean flow Mach number is increased further to 0.3 (see Fig. 10) there is a discrepancy in the educed normalized resistance at the high frequency end of the spectra where a higher order vertical mode is cuton. Recall that the sound source in the upstream hard wall section is assumed to be a plane wave and this assumption breaks down when a higher order mode cuts on in either the vertical or horizontal direction.

The educed normalized reactance spectra for the uniform-structure and the soft segment of the peripherally varying liner is presented in Fig. 11. In the absence of flow (i.e., Mach 0.0) there is very good agreement except at the low frequency end of the spectra where the attenuation is low. The low attenuation also leads to a discrepancy in educed reactance at the low frequency end of the spectra for Mach 0.2 and 0.3. There is also a discrepancy in educed reactance at the highest Mach number (Mach 0.3) at the high frequency end of the spectra where the next higher order vertical duct mode is almost cuton.

One important observation that has not been discussed is that as the flow Mach number is increased for the uniformstructure liner there is some energy scattered into horizontal duct modes. The vertical boundary layer cannot produce this type of mode scattering and the SPL and Mach number are too low for nonlinear effects to be the cause. In addition, this horizontal scattering of acoustic energy did not occur for the uniform-structure liner when the flow was turned off, illustrating that it was not due to horizontal impedance inhomogeneities. Therefore this horizontal scattering of acoustic energy appears to be due to the horizontal boundary layer effects. Thus, the effects of the horizontal boundary layer should be included in future impedance eductions.

\section{Conclusions}

This paper extends the two-dimensional impedance eduction technique presented in earlier papers ${ }^{1,2,4,5}$ to threedimensional sound fields and peripherally varying duct liners. Based upon the results of this study, the following 
conclusions are drawn:

1. When synthesized data (that is based on exact mode theory and known impedance spectra) is used, the current three-dimensional method educes the known impedance spectra to design order.

2. When measured GFIT data is used with the uniform-structure test samples, the three-dimensional theory reproduces the same impedance spectra as the two-dimensional theory except for frequencies corresponding to very low or very high liner attenuation.

3. When the educed impedance of the uniform-structure liner is compared to that of the soft portion of the threesegmented peripherally varying liner, good agreement is generally obtained except for those frequencies corresponding to extremely large attenuation (this was expected).

\section{Acknowledgment}

This work is funded by the Advanced Air Transport Technologies Project of NASA's Advanced Air Vehicles Program.

\section{References}

\footnotetext{
${ }^{1}$ Watson, W. R., Jones, M. G., Tanner, S. E., and Parrott, T. L., "A Finite Element Propagation Model for Extracting Normal Incidence Impedance in Nonprogressive Acoustic Wave Fields," Journal of Computational Physics, Vol. 125, Issue 1, Apr. 1996, pp. 177-186.

${ }^{2}$ Watson, W. R., Jones, M. G., and Parrott, T. L., "Validation of an Impedance Eduction Method in Flow," AIAA Journal, Vol. 37, No. 7, July 1999, pp. 818-824.

${ }^{3}$ Jones, M. G., Watson, W. R., Tracy, M. B., Parrott, T. L., "Comparison of Two Waveguide Methods for Educing Liner Impedance in Grazing Flow." AIAA Journal, Vol. 42, No. 2, Feb. 2004, pp. 232-240.

${ }^{4}$ Jones, M., Watson, W., and Parrott, T., "Benchmark Data for Evaluation of Aeroacoustic Propagation Codes with Grazing Flow." AIAA Paper 2005-2853, May 2005.

${ }^{5}$ Watson, W. R., Jones, M. G., and Parrott, T. L., "Comparison of a Convected Helmholtz and Euler Model for Impedance Eduction in Flow," AIAA Paper 2006-2643, May 2006.

${ }^{6}$ Jones, M. G., Watson, W. R., and Nark, D. M., "Effects of Flow Profile on Educed Acoustic Liner Impedance," AIAA Paper 2010-3763, June 2010.

${ }^{7}$ Armstrong, D. L., Beckemeyer, R. J., and Olsen, F. R., "Impedance Measurements of Acoustic Duct Liners with Grazing Flow," Journal of the Acoustical Society of America, Vol. 55, No. S1, Apr. 1974, p. S59.

${ }^{8}$ Watson, W. R., "A Method for Determining Acoustic Liner Admittance in a Rectangular Duct with Grazing Flow from Experimental Data," NASA TP-2310, July 1984.

${ }^{9}$ Watson, W. R., "A New Method for Determining Acoustic-Liner Admittance in Ducts with Sheared Flow in Two Cross-Sectional Directions," NASA TP-2518, June 1985.

${ }^{10}$ Parrott, T. L., Watson, W. R., and Jones, M. G., "Experimental Validation of a Two-dimensional Shear Flow Model for Determining Acoustic Impedance," NASA TP-2679, May 1987.

${ }^{11}$ Jing, X., Peng, S., and Sun, X., "A Straightforward Method for Wall Impedance Eduction in a Flow Duct," Journal of the Acoustical Society of America, Vol. 124, No. 1, July 2008, pp. 227-234.

${ }^{12}$ Renou, Y. and Auregan Y., "Failure of the Ingard-Myers Boundary Condition for a Lined Duct: An Experimental Investigation," Journal of the Acoustical Society of America, Vol. 130, No. 1, July 2011, pp. 52-60.

${ }^{13}$ Watson, W. R., and Jones, M. G., "A Comparative Study of Four Impedance Eduction Methodologies Using Several Test Liners." AIAA Paper 2013-2274, June 2013.

${ }^{14}$ Watson, W. R., Gerhold, C. H., and Jones, M. G., "Single Mode Theory for Impedance Eduction in Large-Scale Ducts with Grazing Flow, " AIAA Paper-2014-3351, June 2014.

${ }^{15}$ Watson, W. R., and Jones, M. G., "A New Numerical Procedure for Impedance Eduction in Ducts Containing Mean Flow," AIAA Journal, Vol. 49, No. 10, October, 2011, pp. 2109-2122.

${ }^{16}$ Myers, M. K., "On the Acoustic Boundary Condition in the Presence of Flow," Journal Of Sound And Vibrations, Vol. 71, No. 3, Aug. 1980, pp. 429-434.

${ }^{17}$ Watson, W. R., and Lansing, D. L., “A Comparison of Matrix Methods for Calculating Eigenvalues in Acoustically Lined Ducts,” NASA TN D-8186, Mar. 1976.

${ }^{18}$ Stewart, G. W., III, “A Modification of Davidon's Minimization Method to Accept Difference Approximations of Derivatives," Journal of ACM, Vol. 14, No. 1, 1967, pp. 72-83.
} 